\title{
HERMITE INTERPOLATION VISITS ORDINARY TWO-POINT BOUNDARY VALUE PROBLEMS
}

\author{
R. E. GRUNDY ${ }^{1}$
}

(Received 25 August, 2006; revised 24 April, 2007)

\begin{abstract}
This paper is concerned with constructing polynomial solutions to ordinary boundary value problems. A semi-analytic technique using two-point Hermite interpolation is compared with conventional methods via a series of examples and is shown to be generally superior, particularly for problems involving nonlinear equations and/or boundary conditions.
\end{abstract}

2000 Mathematics subject classification: primary 65L10; secondary 65D30.

Keywords and phrases: boundary value problems, Hermite interpolation.

\section{Introduction}

In this paper we introduce a semi-analytic method for constructing polynomial solutions of ordinary boundary value problems using two-point Hermite interpolation. The method has recently been applied to partial differential equations (Grundy [4-6]), however in the present paper we concentrate on the development of the application to ordinary differential equations. With the advent of modern symbolic computational facilities it has become possible to implement many techniques which were hitherto computationally and algebraically inaccessible. Thus an important feature of the paper is the use of the symbolic computational package MAPLE in the process of implementation together with its BVP and IVP library codes as a checking device. The main purpose is to demonstrate the general superiority of our preferred method vis-à-vis conventional methods, particularly for nonlinear and/or singular boundary value problems. This even extends to purely numerical methods which are often difficult to implement for singular problems and also for nonlinear problems with multiple solutions. The structure of the paper is as follows. In Sections 2 and 3 we introduce the idea of two-point Hermite interpolation and its application to ordinary boundary

\footnotetext{
${ }^{1}$ School of Mathematics and Statistics, University of St Andrews, St Andrews, UK; email: reg@st-andrews.ac.uk.

(C) Australian Mathematical Society 2007, Serial-fee code 1446-1811/07
} 
value problems. In Section 4 we include a series of examples to illustrate various advantageous aspects of the method for regular boundary and eigenvalue problems while in Section 5 we show how the method can offer a simple and neat treatment of singular boundary value problems. The results are compared where possible with the BVP software available in the MAPLE library.

\section{Two-point Hermite interpolation}

We first say something about two-point Hermite interpolation [7]. Essentially this is a generalization of interpolation using Taylor polynomials and for that reason Hermite interpolation is sometimes referred to as two-point Taylor interpolation. The idea is to approximate a function $y(x)$ by a polynomial $p(x)$ in which values of $y(x)$ and any number of its derivatives at given points are fitted by the corresponding function values and derivatives of $p(x)$. In this paper we are particularly concerned with fitting function values and derivatives at the two end points of a finite interval, say $[0,1]$, wherein a useful and succinct way of writing a Hermite interpolant $p_{n}(x)$ of degree $2 n+1$ was given for example by Phillips [10] as

$$
p_{n}(x)=\sum_{j=0}^{n}\left\{y^{(j)}(0) q_{j}(x)+(-1)^{j} y^{(j)}(1) q_{j}(1-x)\right\}
$$

where

$$
q_{j}(x)=\frac{x^{j}}{j !}(1-x)^{n+1} \sum_{s=0}^{n-j}\left(\begin{array}{c}
n+s \\
s
\end{array}\right) x^{s}=\frac{Q_{j}(x)}{j !}
$$

so that (2.1) with (2.2) satisfies

$$
y^{(r)}(0)=p_{n}^{(r)}(0), \quad y^{(r)}(1)=p_{n}^{(r)}(1), \quad r=0,1,2 \ldots n,
$$

implying that $p_{n}(x)$ agrees with the appropriately truncated Taylor series for $y(x)$ about $x=0$ and $x=1$.

The error on $[0,1]$ is given by

$$
R_{n}=y(x)-p_{n}(x)=\frac{(-1)^{n+1} x^{n+1}(1-x)^{n+1} y^{(2 n+2)}(\xi)}{(2 n+2) !}
$$

where $0<\xi<1$ and $y^{(2 n+2)}$ is assumed to be continuous.

The Hermite interpolant for $p_{n}(x)$ may converge to $y(x)$ in $[0,1]$ irrespective of whether the intervals of convergence of the constituent series intersect or are disjoint. The important consideration here is whether $R_{n} \rightarrow 0$ as $n \rightarrow \infty$ for all $x$ in $[0,1]$. In the application to the boundary value problems in this paper such convergence with $n$ is always confirmed numerically. 
We observe that (2.1) fits an equal number of derivatives at each end point but it is possible and indeed sometimes desirable to use polynomials which fit different numbers of derivatives at the end points of an interval. There are algorithms for constructing such polynomials and the reader is referred to Davis [2] or Stoer and Bulirsch [12] for further details and references, including error estimates. As an example of a two-point Hermite interpolant we may take $n=2$ so that (2.1) with (2.2) becomes the quintic

$$
\begin{aligned}
p_{2}(x)= & (1-x)^{3}\left(1+3 x+6 x^{2}\right) y(0)+x^{3}\left(10-15 x+6 x^{2}\right) y(1) \\
& +x(1-x)^{3}(1+3 x) y^{\prime}(0)-x^{3}(1-x)(4-3 x) y^{\prime}(1) \\
& +\frac{1}{2} x^{2}(1-x)^{3} y^{\prime \prime}(0)+\frac{1}{2} x^{3}(1-x)^{2} y^{\prime \prime}(1)
\end{aligned}
$$

satisfying

$$
\begin{array}{lll}
p_{2}(0)=y(0), & p_{2}^{\prime}(0)=y^{\prime}(0), & p_{2}^{\prime \prime}(0)=y^{\prime \prime}(0), \\
p_{2}(1)=y(1), & p_{2}^{\prime}(1)=y^{\prime}(1), & p_{2}^{\prime \prime}(1)=y^{\prime \prime}(1) .
\end{array}
$$

Finally we observe that (2.1) can be written directly in terms of the Taylor coefficients $a_{i}$ and $b_{i}$ about $x=0$ and $x=1$ respectively, as

$$
p_{n}(x)=\sum_{j=0}^{n}\left\{a, Q_{j}(x)+(-1)^{j} b_{j} Q_{j}(1-x)\right\} .
$$

\section{Solution of second-order boundary value problems}

We consider the boundary value problem

$$
\begin{aligned}
y^{\prime \prime}+f\left(y, y^{\prime}, x\right) & =0 \\
g_{i}\left(y(0), y(1), y^{\prime}(0), y^{\prime}(1)\right) & =0, \quad i=1,2,
\end{aligned}
$$

where $f, g_{1}, g_{2}$ are in general nonlinear functions of their arguments.

The simple idea behind the use of two-point polynomials is to replace $y(x)$ in problem (3.1)-(3.2), or an alternative formulation of it, by a $p_{n}$ which enables any unknown boundary values or derivatives of $y(x)$ to be computed.

The first step therefore is to construct the $p_{n}$. To do this we need the Taylor coefficients of $y(x)$ at $x=0$ and $x=1$. Using MAPLE throughout we simply insert the series forms

$$
y=a_{0}+a_{1} x+\sum_{i=2}^{\infty} a_{i} x^{i} \quad \text { and } \quad y=b_{0}+b_{1}(x-1)+\sum_{i=2}^{N} b_{i}(x-1)^{i}
$$


into (3.1) and equate coefficients of powers of $x$ and $(x-1)$ respectively. The resulting system of equations can be solved to obtain $a_{i}\left(a_{0}, a_{1}\right)$ and $b_{i}\left(b_{0}, b_{1}\right)$ for all $i \geq 2$. The notation implies that the coefficients depend only on the indicated unknowns $a_{0}$, $a_{1}, b_{0}, b_{1}$. The algebraic manipulations needed for this process for Example 4.1 are included in the MAPLE code in the Appendix.

We are now in a position to construct a $p_{n}(x)$ from (3.3) of the form (2.1) and use it as a replacement in the problem (3.1)-(3.2). Since we have only the four unknowns to compute for any $n$ we only need to generate two equations from this procedure as two equations are already supplied by the boundary conditions (3.2).An obvious way to do this would be to satisfy the equation (3.1) itself at two selected points $x=c_{1}$, $x=c_{2}$ in $[0,1]$ so that the two required equations become

$$
p_{n}^{\prime \prime}\left(c_{i}\right)+f\left\{p_{n}\left(c_{i}\right), p_{n}^{\prime}\left(c_{i}\right), c_{i}\right\}=0, \quad i=1,2 .
$$

An alternative approach is to recast the problem in an integral form before doing the replacement. Extensive computations have shown that this generally provides a more accurate polynomial representation for a given $n$. We therefore use this alternative formulation throughout this paper although we should keep in mind that the procedure based on (3.4) is a viable option and shares many common features with the approach outlined below. Of the many ways we could provide an integral formulation we adopt the following. We first integrate (3.1) to obtain

$$
y^{\prime}(x)-a_{1}+\int_{0}^{x} f\left(y(s), y^{\prime}(s), s\right) d s=0
$$

and again to find

$$
y(x)-a_{0}-x a_{1}+\int_{0}^{x}(x-s) f\left(y(s), y^{\prime}(s), s\right) d s=0
$$

where $a_{0}=y(0)$ and $a_{1}=y^{\prime}(0)$. Putting $x=1$ in (3.5) and (3.6) then gives

$$
b_{1}-a_{1}+\int_{0}^{1} f\left(y(s), y^{\prime}(s), s\right) d s=0
$$

and

$$
b_{0}-a_{0}-a_{1}+\int_{0}^{1}(1-s) f(y(s), y(s), s) d s=0
$$

where $b_{0}=y(1)$ and $b_{1}=y^{\prime}(1)$.

The precise way we make the replacement of $y(x)$ with a $p_{n}(x)$ in (3.7) and (3.8) depends on the nature of $f\left(y, y^{\prime}, x\right)$ and will be explained in the examples which follow. In any event the important point to note is that once this replacement has been made, the equations (3.2), (3.7) and (3.8) constitute the four equations we require to determine the set $\left\{a_{0}, b_{0}, a_{1}, b_{1}\right\}$. As we shall see the fact that the number of 
unknowns is independent of the number of derivatives fitted represents perhaps the most important feature of the method.

We make the following points at this stage.

(i) In the majority of cases where the boundary conditions are simple enough the system of algebraic equations may be reduced a priori to a system in two unknowns, since the boundary condition can be substituted directly into the integral formulations (3.7) and (3.8), which MAPLE can be utilized to solve. Once the unknown pair $\left\{a_{1}, b_{1}\right\}$ is known the required polynomial can be constructed. For the benefit of the reader the entire procedure for Example 4.1 in Section 4 is given as a MAPLE code in the Appendix.

(ii) For higher order equations or a system of first-order equations the number of unknowns will of course increase. For an equation of order $m$ there will be $2 m$ unknowns in the equations corresponding to (3.7) and (3.8) or their equivalent. The boundary conditions will supply $m$ equations so we will need a further $m$ equations to determine the $2 m$ unknowns. As in (i) the boundary conditions may enable us to immediately reduce the number of equations, invariably to $m$.

(iii) As we will see with such a low number of unknowns it is a relatively simple matter to solve the equations even for nonlinear problems. This is particularly true if $n$ is a small integer. Since, as we shall see, the method provides a convergent sequence for the unknowns as $n$ increases, for higher values of $n$ we may use the values provided at a previous iterate as initial guesses for a simple application of Newton's method usually for two equations in two unknowns.

(iv) The method offers a certain amount of flexibility. For example we could choose to satisfy (3.5) and (3.6) at two internal points or we could use alternative integral formulations. The fact remains that whatever strategy we adopt produces a quickly convergent sequence of values of the set $\left\{a_{0}, a_{1}, b_{0}, b_{1}\right\}$ as $n$ increases.

(v) Throughout we assess the accuracy of the procedure by examining the convergence with $n$. Using a symbolic computational facility such as MAPLE, computing the required convergents is not an issue. Where possible we can also run checks on our solutions using shooting with MAPLE IVP codes.

(vi) We compare our method with the method of collocation using Chebyshev polynomials. This is the method used in the NAG library for regular linear problems. The first use of Chebyshev polynomials in the context was apparently in 'the method of selected points' due to Lanczos [8], see also Lanczos [9], Fox and Parker [3] and Picken [11]. To be brief we first construct the finite Chebyshev series

$$
y(x) \equiv Y_{N}(x)=\sum_{k=0}^{N} c_{k} T(k, x)
$$

where the $c_{k}$ are unknown constants and the $T(k, x)$ are the Chebyshev polynomials of order $k$ rescaled to the interval $[0,1]$. We now use collocation which can be used 
in conjunction with the equation itself or with an integrated form. Fox and Parker [3] recommend an integrated form as generally the most accurate and this is what we use here. The twice indefinitely integrated form of (3.1) can be written as

$$
y(x)+\int_{0}^{x} \int_{0}^{s} f\left(y(r), y^{\prime}(r), r\right) d r d s+A x+B=0,
$$

where $A$ and $B$ are arbitrary constants. We now replace $y(x)$ in (3.10) by (3.9) and collocate at the $N+1$ zeros of $T(N+1, x)$ on $[0,1]$ giving $N+1$ equations. A further two equations are provided by requiring that (3.9) satisfies the two boundary conditions (3.2). Thus in total we have $N+3$ equations for the $N+3$ unknowns $\left\{c_{0}, c_{1}, \ldots, c_{N}, A, B\right\}$. For linear equations with linear boundary conditions solving these poses no problems. However any nonlinearities in either the equation or the boundary conditions will pose often insuperable difficulties which are not resolved even if we resort to performing collocation on the equation itself thereby reducing the number of equations and unknowns. In any event the fact that the two-point method only involves at most four unknowns whatever the value of $n$ will in combination with Newton's method, always obviate the difficulty in using (3.9) with (3.10).

Finally in assessing the two methods we observe that we should compare $y_{n}$ with $Y_{2 n+1}$ since they are both of degree $2 n+1$. Although we should remember that in computing $Y_{2 n+1}$ we have to solve a system of $2 n+4$ equations, in general nonlinear, for the $2 n+4$ unknowns.

We now consider a number of examples designed to illustrate the convergence, accuracy, implementation and utility of the method. In what follows the use of bold digits in the tables is intended to give a rough visual indication of the convergence.

\section{Examples}

4.1. A linear problem We first consider the linear problem

$$
y^{\prime \prime}+y \sin (x)=e^{x}, \quad y(0)=1, \quad y(1)=0 .
$$

Here (3.7) and (3.8) become

$$
\begin{aligned}
& 1+b_{1}-a_{1}-e+\int_{0}^{1} y(s) \sin (s) d s=0, \\
& 1-a_{1}-e+\int_{0}^{1}(1-s) y(s) \sin (s) d s=0
\end{aligned}
$$

and the coefficients in (3.3) are $a_{0}=1, a_{2}=1 / 2, a_{3}=0, a_{4}=\left(1-2 a_{1}\right) / 24, \ldots$, $b_{0}=0, b_{2}=e / 2, b_{3}=\left(e-b_{1} \sin (1)\right) / 6, b_{4}=\left(e-2 b_{1} \cos (1)-e \sin (1)\right) / 24, \ldots$ 
TABLE 1. Comparison of the Hermite and Chebyshev methods with the numerical solution for Example 4.1.

\begin{tabular}{|c|c|c|c|c|c|c|}
\hline & $p_{2}$ & $p_{3}$ & $p_{4}$ & $N=4$ & $N=5$ & Numerical \\
\hline$a_{1} \equiv y^{\prime}(0)$ & -1.662609 & -1.662598 & -1.662598 & -1.664480 & -1.662905 & -1.662598 \\
\hline$b_{1} \equiv y^{\prime}(1)$ & -0.039280 & -0.039293 & -0.039293 & -0.035565 & -0.039094 & -0.039293 \\
\hline$x=0.2$ & 0.687732 & 0.687764 & 0.687766 & 0.687805 & 0.687765 & 0.687766 \\
\hline$x=0.5$ & 0.304453 & 0.304559 & 0.304566 & 0.304558 & 0.304559 & 0.304566 \\
\hline$x=0.8$ & 0.058573 & 0.058598 & 0.058599 & 0.058543 & 0.058598 & 0.058599 \\
\hline
\end{tabular}

TABLE 2. Convergence of the Chebyshev coefficients for $N=2$ to 6 using collocation at the zeros of $T(N+1, x)$ and satisfaction of the boundary conditions.

\begin{tabular}{|c|c|c|c|c|c|c|c|}
\hline $\mathrm{N}$ & $c_{0}$ & $c_{1}$ & $c_{2}$ & $c_{3}$ & $c_{4}$ & $c_{5}$ & $c_{6}$ \\
\hline 2 & 0.403388 & -0.5 & 0.096612 & - & - & - & - \\
\hline 3 & 0.402299 & -0.509591 & 0.097701 & 0.009591 & - & - & - \\
\hline 4 & 0.401333 & -0.509525 & 0.097721 & 0.009525 & 0.000946 & - & - \\
\hline 5 & 0.401337 & -0.509454 & 0.097721 & 0.009525 & 0.000942 & -0.000071 & - \\
\hline 6 & 0.401341 & -0.509455 & 0.097721 & 0.009525 & 0.000942 & -0.000071 & -0.000004 \\
\hline
\end{tabular}

$A b$ initio inclusion of the boundary conditions in (4.2)-(4.3) has reduced the number of unknowns to two, namely $\left\{a_{1}, b_{1}\right\}$, which are computed by solving (4.2) and (4.3) with $y(s)$ replaced by a $p_{n}(s)$. A MAPLE code for this example is presented in the Appendix.

The results for $n=2,3,4$ and $N=4,5$ are displayed in Table 1. We can see that there is clear convergence with $n$ to the 'exact' values which are obtained using MAPLE boundary value software. There appears to be little to choose in accuracy between the two methods although the Chebyshev method is more labour intensive as $N$ increases.

4.2. A problem with nonlinear boundary conditions We next consider the same equation as (4.1), namely $y^{\prime \prime}+y \sin (x)=e^{x}$, but this time with the nonlinear boundary conditions

$$
y(1)=\{y(0)\}^{2} \text { and } \quad y^{\prime}(1)=\left\{y^{\prime}(0)\right\}^{2} .
$$

The integral formulation incorporating the boundary conditions now becomes

$$
\begin{aligned}
& 1+a_{1}^{2}-a_{1}-e+\int_{0}^{1} y(s) \sin (s) d s=0, \\
& 2+a_{0}^{2}-a_{0}-a_{1}-e+\int_{0}^{1}(1-s) y(s) \sin (s) d s=0
\end{aligned}
$$


TABLE 3. Results for Example 2 using Hermite interpolating polynomials and the comparison with Chebyshev polynomials with $N=5$.

\begin{tabular}{|c|c|c|c|c|c|c|}
\hline \multicolumn{2}{|c|}{} & $p_{2}$ & $p_{3}$ & $p_{4}$ & $N=5$ & Numerical \\
\hline \multirow{2}{*}{$S_{1}$} & $a_{0} \equiv y(0)$ & 0.247485 & 0.247486 & 0.247486 & 0.247309 & - \\
\cline { 2 - 7 } & $a_{1} \equiv y^{\prime}(0)$ & -0.906862 & -0.906861 & -0.906861 & -0.906910 & - \\
\hline \multirow{2}{*}{$S_{2}$} & $a_{0}$ & 0.762827 & 0.762827 & 0.762827 & 0.762938 & - \\
\cline { 2 - 7 } & $a_{1}$ & -0.815394 & -0.815394 & -0.815393 & -0.815378 & - \\
\hline \multirow{2}{*}{$S_{3}$} & $a_{0}$ & 1.862214 & 1.862228 & 1.862229 & 1.861893 & 1.862229 \\
\cline { 2 - 7 } & $a_{1}$ & 1.301425 & 1.301448 & 1.301448 & 1.301011 & 1.301448 \\
\hline \multirow{2}{*}{$S_{4}$} & $a_{0}$ & -1.179826 & -1.179841 & -1.179841 & -1.179521 & - \\
\cline { 2 - 7 } & $a_{1}$ & 1.841360 & 1.841384 & 1.841384 & 1.840909 & - \\
\hline
\end{tabular}

TABLE 4. A check on the accuracy of the solution by shooting from $x=0$ with $a_{0} \equiv y(0), a_{1} \equiv y^{\prime}(0)$ obtained from $p_{4}$ rounded to six decimal places, for each of the 4 solutions. Here $\Delta_{1}=\left|y(1)-\{y(0)\}^{2}\right|$ and $\Delta_{2}=\left|y^{\prime}(1)-\left\{y^{\prime}(0)\right\}^{2}\right|$.

\begin{tabular}{|c|c|c|}
\hline & $\Delta_{1}$ & $\Delta_{2}$ \\
\hline$S_{1}$ & $1.848 \times 10^{-7}$ & $1.267 \times 10^{-7}$ \\
\hline$S_{2}$ & $5.063 \times 10^{-7}$ & $1.375 \times 10^{-6}$ \\
\hline$S_{3}$ & $8.484 \times 10^{-7}$ & $2.103 \times 10^{-6}$ \\
\hline$S_{4}$ & $7.853 \times 10^{-7}$ & $1.965 \times 10^{-6}$ \\
\hline
\end{tabular}

and the coefficients in (3.3) are $a_{2}=1 / 2, a_{3}=\left(1-a_{0}\right) / 6, a_{4}=\left(1-2 a_{1}\right) / 24$, $\ldots, b_{0}=a_{0}^{2}, b_{1}=a_{1}^{2}, b_{2}=\left\{e-a_{0}^{2} \sin (1)\right\} / 2, b_{3}=\left\{e-a_{0}^{2} \cos (1)-a_{1}^{2} \sin (1)\right\} / 6$, $b_{4}=\left\{a_{0}^{2} \sin ^{2}(1)-2 a_{1}^{2} \cos (1)+a_{0}^{2} \sin (1)+e-e \sin (1)\right\} / 24, \ldots$

Again inclusion of the boundary conditions has reduced the number of unknowns to two, namely $\left\{a_{0}, a_{1}\right\}$. The four solutions to this problem are identified by our method and presented in Table 3. The penultimate column gives the results using Chebyshev polynomials with $N=5$. Clearly the results using two-point approximants are superior. We can also use the MAPLE boundary value code to solve the problem numerically which uses the mid-point method with Richardson extrapolation and is employed throughout the paper; see for example Ascher, Mattheif and Russell [1]. Table 4 presents a check on our results by shooting from $x=0$ with the values of $y(0)$ and $y^{\prime}(0)$ obtained from $p_{4}$. The results are consistent after rounding to 6 decimal places.

4.3. A nonlinear equation Next we consider the nonlinear equation

$$
y^{\prime \prime}+\lambda y^{2}=e^{x}
$$

with boundary conditions

$$
y(0)=1, \quad y(1)=2 \text {. }
$$


TABLE 5. Results for $y^{\prime \prime}+\lambda y^{2}=e^{x}, y(0)=1, y(1)=2$ with $\lambda=1$. Collocation with Chebyshev polynomials and the MAPLE numerical code fail to locate $S_{2}$ due to root-finding problems. The numerical solution for $S_{2}$ in the table is found by iterative shooting from $x=0$ with the initial guess for $y^{\prime}(0)=p_{6}^{\prime}(0)$. For $S_{2}$ the values for the pair $\left\{a_{1}, b_{1}\right\}$ at each value of $n>6$ are found using Newton's method with the initial guesses provided by the previous value of $n$.

\begin{tabular}{|c|c|c|c|c|c|c|c|c|}
\hline \multicolumn{2}{|c|}{} & $p_{2}$ & $p_{3}$ & $p_{4}$ & $p_{5}$ & $p_{6}$ & $N=5$ & Numerical \\
\hline \multirow{4}{*}{$s_{1}$} & $a_{1}=y^{\prime}(0)$ & 1.301093 & 1.301064 & 1.301052 & 1.301052 & 1.301052 & 1.301472 & 1.301052 \\
\cline { 2 - 9 } & $b_{1} \equiv y^{\prime}(1)$ & 0.452402 & 0.452410 & 0.452522 & 0.452422 & 0.452422 & 0.452654 & 0.452422 \\
\cline { 2 - 9 } & $x=0.2$ & 1.257966 & 1.257933 & 1.257929 & 1.257928 & 1.257928 & 1.257930 & 1.257928 \\
\cline { 2 - 9 } & $x=0.5$ & 1.612581 & 1.612553 & 1.612536 & 1.612536 & 1.612536 & 1.612540 & 1.612536 \\
\cline { 2 - 9 } & $x=0.8$ & 1.883145 & 1.883159 & 1.883155 & 1.883155 & 1.883155 & 1.883155 & 1.883155 \\
\hline & $p_{4}$ & $p_{6}$ & $p_{8}$ & $p_{10}$ & $p_{12}$ & & \\
\hline \multirow{4}{*}{$S_{2}$} & $a_{1}=y^{\prime}(0)$ & 23.930284 & 23.707665 & 23.695173 & 23.694518 & 23.694486 & - & 23.694465 \\
\cline { 2 - 9 } & $b_{1} \equiv y^{\prime}(0)$ & -23.555406 & -23.321257 & -23.308087 & -23.307395 & -23.307361 & - & -23.307340 \\
\cline { 2 - 9 } & $x=0.2$ & 5.645258 & 5.604082 & 5.604268 & 5.604145 & 5.604139 & - & 5.604135 \\
\cline { 2 - 9 } & $x=0.5$ & 9.578566 & 9.545708 & 9.545783 & 9.545721 & 9.545718 & - & 9.545713 \\
\cline { 2 - 9 } & $x=0.8$ & 6.482310 & 6.439980 & 6.440172 & 6.440044 & 6.440038 & - & 6.440038 \\
\hline
\end{tabular}

Here (3.7) and (3.8) become

$$
\begin{aligned}
& F\left(a_{1}, b_{1}, \lambda\right) \equiv 1+b_{1}-a_{1}-e+\lambda \int_{0}^{1} y^{2}(s) d s=0, \\
& G\left(a_{1}, b_{1}, \lambda\right) \equiv 1-a_{1}-e+\lambda \int_{0}^{1}(1-s) y^{2}(s) d s=0
\end{aligned} .
$$

and the coefficients in (3.3) are $a_{0}=1, a_{2}=(1-\lambda) / 2, a_{3}=\left(1-2 \lambda a_{1}\right) / 6$, $a_{4}=\left(1-2 \lambda a_{1}^{2}-2 \lambda+2 \lambda^{2}\right) / 24, \ldots, b_{0}=2, b_{2}=(e-4 \lambda) / 2, b_{3}=\left(e-4 \lambda b_{1}\right) / 6$, $b_{4}=\left(e+16 \lambda^{2}-4 e \lambda-2 \lambda b_{1}^{2}\right) / 24, \ldots$

Taking $\lambda=1$ we obtain the results presented in Table 5. Only two real solutions are identified for each value of $n$ represented by the two solutions to the problem for this value of $\lambda$. The MAPLE boundary value code only gives the lower solution. The 'exact' solution for the upper root is obtained by iterative-shooting with the initial estimate for $y^{\prime}(0)$ given by $p_{6}$ which illustrates the utility of the two-point method in combination with shooting techniques. As a further refinement we could use the resulting computed values of $\left\{a_{1}, b_{1}\right\}$ to compute a final two-point polynomial for the upper solution.

The results using the Chebyshev series for $N=5$ are also displayed in Table 5. The method is less successful than the two-point option especially for the upper solution where the method fails for $N=4$ and $N=5$ due to root-finding problems. Remember that to obtain a degree 13 polynomial equivalent to $p_{6}$ using Chebyshev polynomials would require us to solve a nonlinear system in 16 unknowns.

It is appropriate at this point to say something about root-finding. We have used the MAPLE solve to locate roots. Where this fails for the two-point method we can if 
TABLE 6. Computation of the threshold value $\lambda=\lambda^{*}$.

\begin{tabular}{|c|c|c|c|c|c|}
\hline & $p_{2}$ & $p_{3}$ & $p_{4}$ & $p_{5}$ & $p_{6}$ \\
\hline$a_{1} \equiv y^{\prime}(0)$ & 5.5009 & 5.1909 & 5.1373 & 5.1279 & 5.1264 \\
\hline$b_{1} \equiv y^{\prime}(1)$ & -4.6993 & -4.3336 & -4.2712 & -4.2603 & -4.2586 \\
\hline$\lambda \equiv \lambda^{*}$ & -1.8503 & 1.8170 & 1.8125 & 1.8119 & 1.8119 \\
\hline
\end{tabular}

necessary use a simple user-provided two-variable Newton's method code using the $n-1$ estimate as a starting value. The problem is less tractable if solve fails for the Chebyshev method since in that event we have to use fsolve which by default only gives one root. To locate the second root we would have to give bounds for each of the $N+3$ unknowns or alternatively give initial estimates for a user-provided code for Newton's method in $N+3$ unknowns. Either option would require the use of the two-point method to provide the starting values making the Chebyshev method itself somewhat redundant.

We continue to analyze this problem using Hermite interpolation. If we repeat the calculations for $\lambda=2$ we find that there are no real roots for $a_{1}$ and $b_{1}$. This suggests that there exists a $\lambda=\lambda^{*}$ such that for $\lambda>\lambda^{*}$ there are no solutions of the boundary value problem while for $\lambda<\lambda^{*}$ there are two. This of course is a well-known feature of the problem. What we do now is to compute the threshold value $\lambda^{*}$ using our two-point method. Essentially this involves finding a double root of (4.4) and (4.5) for $\left(a_{1}, b_{1}\right)$. Thus we have to solve (4.4) and (4.5) together with

$$
\frac{\partial F}{\partial a_{1}} \frac{\partial G}{\partial b_{1}}-\frac{\partial G}{\partial a_{1}} \frac{\partial F}{\partial b_{1}}=0
$$

for the unknowns $a_{1}, b_{1}$ and $\lambda$. The results for $n=2$ to 6 are shown in Table 6 rounding to 4 decimal places.

We thus conclude that $\lambda^{*}=1.8119$ to 4 decimal places. We note that there is no difficulty in taking higher values of $n$ if we wished to refine this value. By comparison an equivalent calculation with $N=13$ using Chebyshev polynomials would require us to solve 17 nonlinear equations in 17 unknowns.

4.4. Variations on a theme This last nonlinear example illustrates another feature of the two-point method which has general application to equations of the following type. Consider the problem

$$
\begin{aligned}
& y^{\prime \prime}+\sin (y)=e^{x}, \\
& y(0)=1 \text { and } y(1)=2 .
\end{aligned}
$$

The difficulty with this example is that it is not in general possible to perform the integrations involving $\sin \left(p_{n}(x)\right)$ when using (3.7) and (3.8). We could of course 
TABLE 7. Results for Example 4.4 together with the comparison with Chebyshev polynomials and the numerical solution.

\begin{tabular}{|c|c|c|c|c|c|c|c|}
\hline & $\left(p_{2}, q_{2}\right)$ & $\left(p_{3}, q_{3}\right)$ & $\left(p_{4}, q_{4}\right)$ & $\left(p_{5}, q_{5}\right)$ & $N=4$ & $N=5$ & Numerical \\
\hline$a_{1} \equiv y^{\prime}(0)$ & 0.749240 & 0.749329 & 0.749359 & 0.749355 & 0.7550700 & 0.749303 & 0.749355 \\
\hline$b_{1} \equiv y^{\prime}(1)$ & 1.515489 & 1.515410 & 1.515379 & 1.515382 & 1.517778 & 1.515515 & 1.515382 \\
\hline$x=0.2$ & 1.153953 & 1.153925 & 1.153932 & 1.153931 & 1.154223 & 1.153932 & 1.153931 \\
\hline$x=0.5$ & 1.411016 & 1.410858 & 1.410871 & 1.410870 & 1.410713 & 1.410936 & 1.410870 \\
\hline$x=0.8$ & 1.729016 & 1.728977 & 1.728984 & 1.728983 & 1.728449 & 1.728972 & 1.728983 \\
\hline
\end{tabular}

consider using alternative strategies along the lines of (3.4). However we choose to continue with the integral form and replace $\sin \left(p_{n}(x)\right)$ itself by a two-point polynomial $q_{n}(x)$ in (3.7) and (3.8). Thus using (3.3) we can write

and

$$
\sin (y(x))=\sum_{j=0}^{M} A_{j}\left(a_{0}, a_{1}\right) x^{j}
$$

$$
\sin (y(x))=\sum_{j=0}^{M} B_{j}\left(b_{0}, b_{1}\right)(x-1)^{j}
$$

from which we can construct the $q_{n}(x)$. Hence (3.7) and (3.8) become

and

$$
b_{1}-a_{1}-e+1+\int_{0}^{1} q_{n}(s) d s=0
$$

$$
3-a_{1}-e+\int_{0}^{1}(1-s) q_{n}(s) d s=0 .
$$

The results are presented in Table 7 where the numerical check is provided by the MAPLE boundary value code.

The only practical way to use Chebyshev polynomials here is to use collocation on the equation directly. From (3.7) we then have

$$
\frac{d^{2}\left\{\left(Y_{N}(x)\right)\right\}}{d x^{2}}+\sin \left\{Y_{N}(x)\right\}-e^{x}=0
$$

and collocate at the $N-1$ zeros of $T(N-1, x)$ as well as satisfying the 2 boundary conditions giving the required number of equations for the $N+1$ unknowns. The equations arising from (4.6) are transcendental which in general may introduce complications in root-finding where there are multiple solutions although not in this case since the solution is unique. The results are displayed in Table 7.

To summarize thus far we have shown how we can use two-point Hermite interpolation to construct polynomial solutions to two-point boundary value problems. In 
general the method appears to be superior to other methods in terms of accuracy and the ability to consistently identify multiple solutions of nonlinear problems where even numerical methods are problematical. Where feasible we have checked our results with those obtained from MAPLE boundary value or initial value codes. In the next section we consider a type of problem where numerical integration may not always be available to us and the Hermite method becomes a preferred option.

\section{Singular boundary value problems}

An important problem in many applications is to find regular solutions to singular boundary value problems - an obvious example is the solution of problems with cylindrical or spherical symmetry in $r \geq 0$. We shall show in this section that two-point interpolation is particularly adept at analyzing such problems since it deals directly with derivatives. We illustrate the simplicity and effectiveness of the technique by three examples which serve to demonstrate that the method succeeds when even numerical integration poses difficulties. In what follows although we deal with linear differential equations, this is no restriction on the method. Singularities at one or both end points of the interval $[0,1]$ may be involved and we proceed in all cases via an integral formulation of the problems.

\subsection{A singular boundary value problem with nonlinear boundary conditions} We use this first example to show not only how we can treat singular problems but also to illustrate how much progress can sometimes be made analytically using the method. We consider the family of linear boundary value problems

$$
x y^{\prime \prime}+a y^{\prime}+\left(b x^{2}+c x+d\right) y=0, \quad a>1,
$$

where we require $y(x)$ to be regular at $x=0$ and

$$
y(1)=k_{1}\{y(0)\}^{2}+k_{2} .
$$

Here $a, b, c, d, k_{1}, k_{2}$ are given constants.

We observe that (5.1) has a regular singularity at $x=0$, with indices 0 and $1-a$, and is regular at $x=1$. We first construct the series for the required solution about $x=0$ and $x=1$. Using (5.1) the solution which satisfies regularity at $x=0$ can be written as

$$
y(x)=\sum_{i=0}^{\infty} a_{i} x^{i}
$$

where $a_{1}=-a_{0} d / a, a_{2}=\left(d^{2}-a c\right) a_{0} / 2 a(a+1), \ldots$. 
TABLE 8. Convergence check for $\alpha(n), \beta(n)$ and $k^{*}(n)$ with $a=3 / 2, b=2, d=-1, c=1, k_{1}=-1$, $k_{2}=1 / 2$.

\begin{tabular}{|c|c|c|c|c|}
\hline & $p_{2}$ & $p_{3}$ & $p_{4}$ & $p_{5}$ \\
\hline$\alpha(n)$ & 1.284328 & 1.284241 & 1.284238 & 1.284238 \\
\hline$\beta(n)$ & -0.421049 & -0.420963 & -0.420960 & -0.420960 \\
\hline$k^{*}(n)$ & 0.412375 & 0.412319 & 0.412317 & 0.412317 \\
\hline
\end{tabular}

The solution can be expanded about $x=1$ as

$$
y(x)=b_{0}+\sum_{i=1}^{\infty} b_{i}(x-1)^{l},
$$

where $b_{2}=-\left\{a b_{1}+(b+c+d) b_{0}\right\} / 2, \ldots$. Thus using (5.3) and (5.4) we can construct any $p_{n}$ which eliminates the singularity at the origin. Assuming regularity, integral formulations can be obtained by integrating (5.1) once and applying the boundary conditions to obtain

$$
b_{1}+(a-1)\left(b_{0}-a_{0}\right)+\int_{0}^{1}\left(b s^{2}+c s+d\right) y(s) d s=0
$$

and integrating (5.1) twice and applying the boundary conditions to give

$$
b_{0}-(a-1) a_{0}+\int_{0}^{1}\left\{(1-s)\left(b s^{2}+c s+d\right)+(a-2)\right\} y(s) d s=0 .
$$

We now replace $y(s)$ with a $p_{n}(s)$ and perform the integrations. By eliminating $b_{0}$ via the boundary condition (5.2), namely $b_{0}=k_{1} a_{0}^{2}+k_{2}$, for each $n$ we can obtain two equations for the unknown pair $\left\{a_{0}, b_{1}\right\}$ of the form

$$
a_{0}^{2}-\frac{\alpha(n)}{k_{1}} a_{0}+\frac{k_{2}}{k_{1}}=0 \quad \text { and } \quad b_{1}=\beta(n) a_{0} .
$$

Thus we have two solutions, one solution or no solutions to the problem according to whether $k_{1} k_{2}<k^{*}(n), k_{1} k_{2}=k^{*}(n)$ or $k_{1} k_{2}>k^{*}(n)$, where $k^{*}(n)=\alpha(n)^{2} / 4$. The values of $\alpha(n), \beta(n)$ and $k^{*}(n)$ rounded to 6 decimal places are displayed in Table 8 .

The MAPLE boundary value code appears to fail for this problem but we can check our solution by shooting from $x=0$ with $y(0)=a_{0}\left(p_{6}\right), y^{\prime}(0)=-a_{0}\left(p_{6}\right) d / a$ where

$$
a_{0}\left(p_{6}\right)=\frac{\left\{\alpha(6) \pm \sqrt{\alpha^{2}(6)-4 k_{1} k_{2}}\right\}}{k_{1}} .
$$

For example with the values of $k_{1}$ and $k_{2}$ in Table 8 we have $a_{0}\left(p_{6}\right)=-1.59727178$ or 0.31303377 and the boundary condition for each solution becomes

$$
y(1)-k_{1}\{y(0)\}^{2}-k_{2}=9.44 \times 10^{-9} \text { and } 6.44 \times 10^{-9},
$$

respectively, which completes the check. 
TABLE 9. Convergence of the first three eigenvalues for Example 5.2.

\begin{tabular}{|c|c|c|c|c|c|c|}
\hline & $p_{2}$ & $p_{4}$ & $p_{6}$ & $p_{8}$ & $p_{10}$ & $p_{12}$ \\
\hline$\lambda_{0}$ & 4.368604 & 4.367012 & 4.367011 & 4.367011 & 4.367011 & 4.367011 \\
\hline$b_{1} \equiv y^{\prime}(1)$ & -0.844876 & -0.845054 & -0.845054 & -0.845054 & -0.845054 & -0.845054 \\
\hline$\lambda_{1}$ & $\mathbf{3 3 . 3 5 5 7 7 3}$ & $\mathbf{3 0 . 8 1 1 8 8 7}$ & 30.790315 & 30.790273 & 30.790273 & 30.790273 \\
\hline$b_{1}$ & $\mathbf{1 . 5 1 1 1 4 8}$ & 1.181181 & 1.177941 & 1.177934 & 1.177934 & 1.177934 \\
\hline$\lambda_{2}$ & $\mathbf{1 3 1 . 0 0 7 8 1 7}$ & $\mathbf{7 8 . 7 4 5 5 1 4}$ & 77.282047 & 77.261002 & 77.260899 & 77.260899 \\
\hline$b_{1}$ & -1.164285 & -1.346719 & -1.369007 & -1.369479 & -1.369481 & -1.369481 \\
\hline
\end{tabular}

5.2. A singular eigenvalue problem We now include a singular eigenvalue problem related to (5.1). We are required to solve

$$
x y^{\prime \prime}+a y^{\prime}+\left(b x^{2}+\lambda x+d\right) y=0, \quad a>1
$$

and compute $\lambda$ subject to $y(0)=1, y(1)=0$ and regularity at $x=0$ with $a, b$ and $d$ given. Here the solution regular at $x=0$ has the form $y(x)=\sum_{i=0}^{\infty} a_{i} x^{i}$, where

$$
a_{0}=1, \quad a_{1}=-\frac{d}{a}, \quad a_{2}=-\frac{\left(d^{2}-\lambda a\right)}{2 a(1+a)}, \quad a_{3}=\frac{-\left\{2 b a(a+1)-\lambda d(2+3 a)+d^{3}\right\}}{a(a+1)(a+2)}, \ldots,
$$

while the solution satisfying the boundary condition at $x=1$ has the form

$$
\begin{gathered}
y(x)=\sum_{i=1}^{\infty} b_{i}(x-1)^{i}, \quad \text { where } \\
b_{2}=-a b_{1} / 2, \quad b_{3}=b_{1}\{a(a+1)-(d+b)-\lambda\} / 6, \ldots
\end{gathered}
$$

Here the integral forms of choice are given by

$$
b_{1}+1-a+\int_{0}^{1}\left(b s^{2}+\lambda s+d\right) y(s) d s=0
$$

and

$$
1-a+\int_{0}^{1}\left\{a-2+\left(b s^{2}+\lambda s+d\right)(1-s)\right\} y(s) d s=0 .
$$

Replacing $y(s)$ by a $p_{n}(s)$ and solving for $b_{1}$ and $\lambda$ yields the results in Table 9 where we have taken $a=3 / 2, b=1$ and $d=1$. The equation for $n=m$ gives estimates for the $m+1$ pairs $\left\{b_{1}, \lambda\right\}$ corresponding to the first $m+1$ eigenvalues and eigenfunctions and we check on convergence by taking values for the first three such pairs.

We can provide a check for these values by shooting from $x=0$ with $y(0)=1$ and $y^{\prime}(0)=-d / a$ to simulate regularity. Taking the values of $\lambda$ from the last column of Table 9 the results of this exercise are displayed in Table 10 for the first three eigenfunctions. 
TABLE 10. Check on the first three eigenvalues by shooting from $x=0$ with the values obtained from the last column in Table 9 with $a=3 / 2, b=1$ and $d=1$.

\begin{tabular}{|c|c|c|}
\hline$\lambda$ & $y(1)$ & $y^{\prime}(1)$ \\
\hline 4.367011 & $-7.17 \times 10^{-8}$ & -0.845054 \\
\hline 30.790273 & $1.96 \times 10^{-7}$ & 1.177934 \\
\hline 77.260899 & $-1.83 \times 10^{-7}$ & -1.369482 \\
\hline
\end{tabular}

5.3. A singular eigenvalue problem arising in the large-time solution of the inviscid Proudman-Johnson equation As a final example we consider the singular eigenvalue problem

$$
\begin{gathered}
\sin (\beta x) y^{\prime \prime}+\beta\{1-2 \cos (\beta x)\} y^{\prime}-\beta^{2} \sin (\beta x) y-K(\beta, \lambda) \\
+\frac{\beta^{3}(1-x) \sin (\beta x)}{\sin (\beta)}+\frac{\beta^{2} \cos (\beta x)}{\sin (\beta)}=0,
\end{gathered}
$$

which has to be solved subject to

$$
y(0)=\beta / \sin (\beta), \quad y(1)=0, \quad y^{\prime}(1)=\lambda
$$

and regularity at $x=0$. Here $0<\beta<\pi$ is given,

$$
K(\beta, \lambda)=2 \beta^{2} \sin (\beta)+\beta^{2} \cot (\beta)+\lambda \beta\{1-2 \cos (\beta)\}
$$

and $\lambda$ is to be found. This example arises in constructing large-time solutions of the inviscid Proudman-Johnson equation (Grundy [6]).

The difficulty here is the logarithmic singularity at $x=0$ in the complementary function so that its behaviour there is

$$
\begin{aligned}
C_{1} x^{2} & \left\{1-\frac{1}{12} \beta^{4} x^{2}+\cdots\right\} \\
& +C_{2}\left\{\ln (x)\left[\beta^{4} x^{2}-\frac{1}{12} \beta^{8} x^{4}+\cdots\right]+2-\frac{5}{6} \beta^{4} x^{2}+\cdots\right\}
\end{aligned}
$$

and we are required to find a global solution for $y(x)$ for which the logarithmic behaviour is absent. It is of course virtually impossible to obtain such a precise representation of the solution by purely numerical means and with this in mind we present this example to show in general how problems involving logarithms can be dealt with using our two-point method.

We first eliminate the offending behaviour by putting $C_{2}=0$ in (5.3). In that event the series for the regular solution of (5.5) about $x=0$ can be written as

$$
y=\sum_{i=0}^{\infty} a_{i} x^{i}
$$


where

$$
\begin{aligned}
& a_{0}=\frac{\beta}{\sin (\beta)}, \quad a_{1}=\frac{\{\lambda \sin \beta(2 \cos (\beta)-1)+\beta(2 \cos (\beta)+1)(\cos (\beta)-1)\}}{\sin (\beta)}, \\
& a_{3}=\frac{\beta^{3}}{\sin (\beta)}, \quad a_{4}=-\frac{\beta^{2} a_{2}}{12}, \\
& a_{5}=-\frac{\beta^{4}\left\{2 \lambda \sin (\beta)(2 \cos (\beta)-1)+\beta\left(4 \cos ^{2}(\beta)-2 \cos (\beta)+15\right)\right\}}{360 \sin (\beta)}, \\
& a_{6}=\frac{\beta^{4} a_{2}}{360}, \ldots
\end{aligned}
$$

and $a_{2}$ is an unknown constant. At $x=1$ we have, from (5.5) and the boundary conditions, the series

$$
y(x)=\sum_{i=0}^{\infty} b_{l}(x-1)^{i}
$$

where

$$
\begin{aligned}
& b_{0}=0, \quad b_{1}=\lambda, \quad b_{2}=\beta^{2}, \quad b_{3}=\frac{\beta^{2}\{2 \beta \cos (\beta)-\lambda \sin (\beta)\}}{6}, \\
& b_{4}=\frac{\beta^{2}\left\{\lambda \sin (\beta)+\beta\left(4 \cos ^{2}(\beta)+\cos (\beta)-4\right\}\right.}{24(1-\cos (\beta))}, \ldots
\end{aligned}
$$

We now follow the general procedure and construct two-point Hermite interpolants $p_{n}$ which together with appropriate integral forms can be used to obtain and solve the two linear equations for the unknown pair $\left\{\lambda, a_{2}\right\}$ for each $p_{n}$. The results are set out in Table 11.

We have been unable to solve the problem numerically for $\beta^{2}>6$ so a full comparison is not possible but the check for other values of $\beta$ is convincing. We observe however that if we examine $a_{1}(\lambda)$ using (5.6) and the numerical entries in Table 11 for $\lambda$ we may spot the formula $a_{1}=-\beta / \sin (\beta)$ and hence, from the expression for $a_{1}$, the formula $\lambda=-\beta \cot (\beta)$ as the reader can readily check.

If we wanted a purely numerical solution to the problem one could conceivably try shooting from $x=1$ with $y(1)=0$ and $y^{\prime}(1)=\lambda$ and iterate on $\lambda$ to hit

$$
\begin{aligned}
y(0) & =\beta / \sin (\beta) \text { and } \\
y^{\prime}(0) & =\{\lambda \sin (\beta)[2 \cos (\beta)-1]+\beta[2 \cos (\beta)+1][\cos (\beta)-1]\} / \sin (\beta) .
\end{aligned}
$$

Even with an a priori estimate of $\lambda$ provided by Table 11 this is not an easy task and the procedure becomes increasingly unreliable as $\beta \uparrow \pi$; in fact for $\beta=\sqrt{9.8}$ the value for $y(0)$ is in error by about $40 \%$. We note that shooting from $x=0$ is not an option due to the singularity there. 


\begin{tabular}{|c|c|c|c|c|c|c|c|c|c|c|c|c|c|c|c|c|c|c|c|c|}
\hline 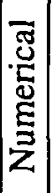 & 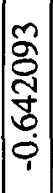 & 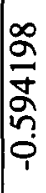 & 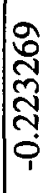 & 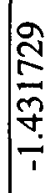 & 先 & 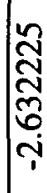 & $\frac{n}{n} \frac{n}{n}$ & $\begin{array}{l}\overline{8} \\
\text { g } \\
\text { m }\end{array}$ & $\mid \begin{array}{l}\stackrel{2}{\infty} \\
\stackrel{\sim}{*} \\
\stackrel{\sim}{r}\end{array}$ & 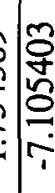 & 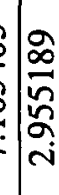 & $\mid \begin{array}{l}N \\
n \\
n \\
n \\
\end{array}$ & 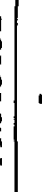 & ' & 1 & 1 & 1 & 1 & I & 1 \\
\hline$\stackrel{\infty}{\infty}$ & & & & & & & & & & & & & 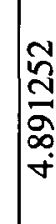 & 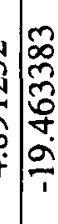 & 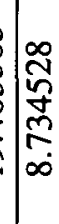 & 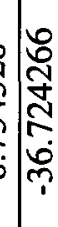 & $\mid \begin{array}{l}\infty \\
n \\
n \\
\vdots \\
0 \\
\end{array}$ & 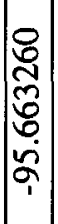 & 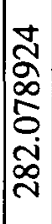 & 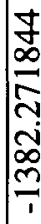 \\
\hline o & & & & & & & $\frac{n}{n}$ & $\begin{array}{l}\overline{8} \\
\text { g } \\
\text { m }\end{array}$ & $\left\{\begin{array}{l}2 \\
\infty \\
\sim \\
\sim \\
\end{array}\right.$ & 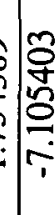 & $\left\{\begin{array}{l}\infty \\
\frac{\infty}{n} \\
\sigma \\
\sigma \\
\sim\end{array}\right.$ & $\mid \begin{array}{l}\frac{n}{n} \\
\frac{n}{n} \\
\frac{n}{1}\end{array}$ & 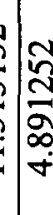 & 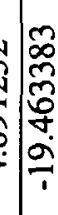 & $\mid \begin{array}{l}\infty \\
\underset{N}{n} \\
\underset{\infty}{\infty} \\
\infty \\
\infty\end{array}$ & 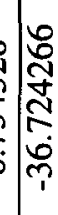 & 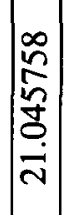 & 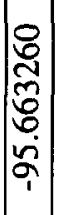 & 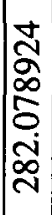 & 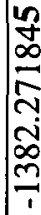 \\
\hline 2 & 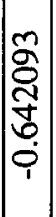 & 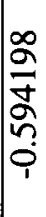 & 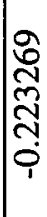 & 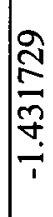 & 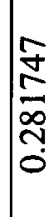 & 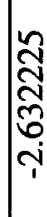 & $\begin{array}{l}\frac{n}{n} \\
\tilde{n} \\
\sigma \\
0\end{array}$ & $\begin{array}{l}\overline{8} \\
8 \\
\text { g } \\
\text { y }\end{array}$ & $\left\{\begin{array}{l}2 \\
\infty \\
\sim \\
\sim \\
\sim\end{array}\right.$ & 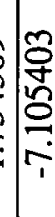 & $\begin{array}{l}\Omega \\
\frac{\infty}{n} \\
2 \\
\sim \\
\sim\end{array}$ & $\mid \frac{n}{n}$ & \begin{tabular}{l}
$\tilde{N}$ \\
\multirow{2}{2}{} \\
$\infty$ \\
$\sigma$
\end{tabular} & 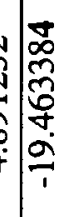 & $\begin{array}{l}\infty \\
\stackrel{\infty}{N} \\
\stackrel{\sim}{N} \\
\stackrel{\infty}{N} \\
\infty\end{array}$ & : & $\mid \begin{array}{l}n \\
n \\
n \\
0 \\
\vec{n}\end{array}$ & 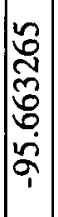 & 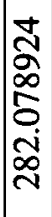 & 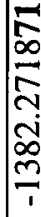 \\
\hline 2 & 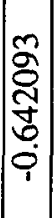 & 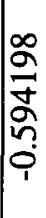 & 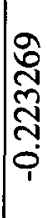 & 尽 & 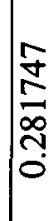 & 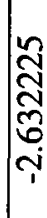 & $\frac{n}{n} \frac{n}{n}$ & $\begin{array}{l}8 \\
8 \\
2 \\
2 \\
\text { ग }\end{array}$ & $\mid \begin{array}{l}\infty \\
\infty \\
\stackrel{\infty}{*} \\
\sim \\
\sim\end{array}$ & 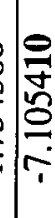 & 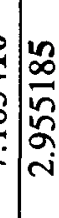 & 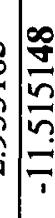 & $\frac{8}{2}$ & 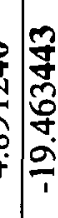 & $\left\{\begin{array}{l}\infty \\
\frac{\infty}{8} \\
\frac{\infty}{2} \\
\infty\end{array}\right.$ & 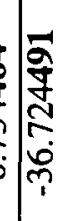 & 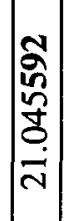 & 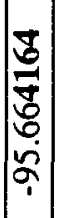 & 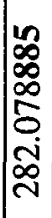 & 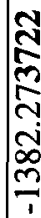 \\
\hline$\dot{Q}$ & 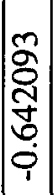 & 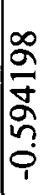 & 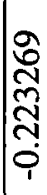 & 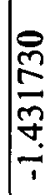 & 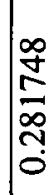 & 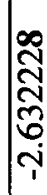 & $\begin{array}{l}\bar{n} \\
\tilde{n} \\
\tilde{n} \\
\sigma \\
0\end{array}$ & 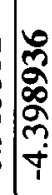 & $\mid$\begin{tabular}{l}
$\tilde{N}$ \\
\multirow{2}{*}{} \\
$\sim$
\end{tabular} & 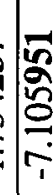 & 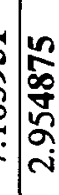 & $\left\{\begin{array}{l}\sqrt[6]{n} \\
\sqrt{n} \\
\frac{7}{2}\end{array}\right.$ & $\frac{a}{\infty}$ & 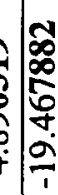 & $\begin{array}{l}\infty \\
\infty \\
\frac{\pi}{m} \\
\frac{\pi}{\infty} \\
\infty\end{array}$ & 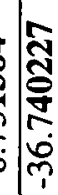 & 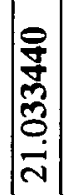 & $\left|\begin{array}{l}\infty \\
\tilde{n} \\
\infty \\
\tilde{N} \\
\tilde{n} \\
\tilde{\gamma}\end{array}\right|$ & 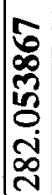 & 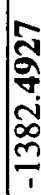 \\
\hline
\end{tabular}




\section{Summary}

In this paper we have presented a semi-analytic technique using two-point Hermite interpolation to construct global polynomial solutions to ordinary boundary value problems with particular emphasis on the use of a symbolic computational facility such as MAPLE. This particular language has been used for the author's convenience but the algorithms could be easily adapted to other symbolic algebraic languages. The advent of such resources has made it possible to access procedures which were formerly considered unprofitable-we believe the use of Hermite interpolation is one such procedure. We have used examples to explain the method and also to highlight the advantages the method has over others such as the classic and still current collocation methods and in some cases numerical integration itself. A prescribed accuracy of the method is attained by examining the convergence with $n$. The main feature is that the number of unknowns we need to find is independent of $n$. This is in contrast to more orthodox collocation where the number of unknown coefficients increases with $n$. This makes root finding for the coefficients an easier task particularly for nonlinear problems with multiple solutions, as well as providing an analytic approximation in the form of a polynomial. The technique may also be used to specify accurate initial estimates for unknown parameters in IVP methods such as shooting. For singular problems any spurious behaviour can be eliminated directly so that use of the method is a practical option even when numerical integration fails or is awkward to apply.

\section{Appendix}

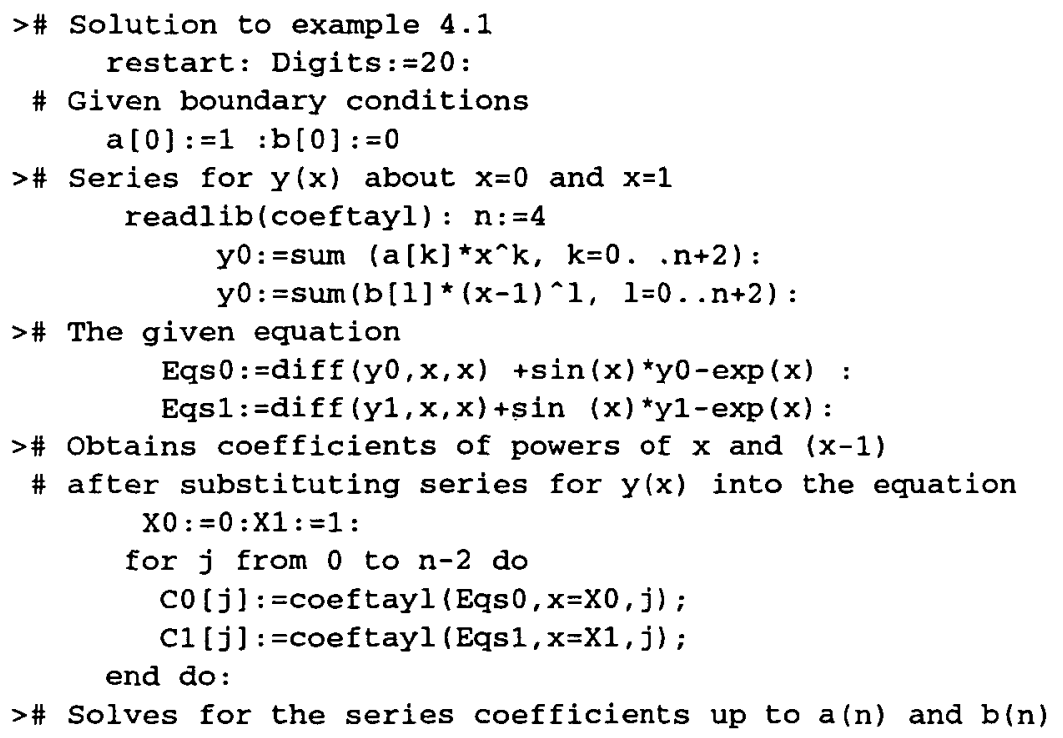


\# in terms of the two unknowns $a(1)$ and $b(1)$

for $i$ from 0 to $n-2$ do

So: =solve $(\{\mathrm{C} 0[i]\},\{a[i+2]\})$ :

S1: =solve $(\{C 1[i]\},\{b[i+2]\}):$

$a[i+2]:=\operatorname{rhs}(S 0[1]) ; b[i+2]:=\operatorname{rhs}(s 1[1]) ;$

$>$ \# Printout of series coefficients as as (n) and bs ( $n$ )

for $i 2$ from 0 to $n$ do

as [i2]:=a[i2];

bs [i2]:=b[i2] ;

end do;

$$
\begin{aligned}
a s_{0} & :=1, \quad a s_{1}:=a_{1}, \quad a s_{2}:=\frac{1}{2}, \quad a s_{3}:=0, \quad a s_{4}:=\frac{1}{24}-\frac{1}{12} a_{1}, \\
b s_{0} & :=0, \quad b s_{1}:=1, \quad b s_{2}:=\frac{1}{2} \mathbf{e}, \quad b s_{3}:=-\frac{1}{6} \sin (1) b_{1}+\frac{1}{6} \mathbf{e}, \\
b s_{4} & :=-\frac{1}{12} \cos (1) b_{1}-\frac{1}{124} \sin (1) \mathbf{e}+\frac{1}{24} \mathbf{e} .
\end{aligned}
$$

$>$ \# Constructs the $Q$ polynomials

for $j$ from 0 to $n$ do

$Q:=\left(s^{\wedge} j^{\star}(1-s)^{\wedge}(n+1)^{\star} \operatorname{sum}(\right.$ (factorial $(n+r))$

(factorial $(r) *$ factorial $\left.(n))^{*} s^{\wedge} r, r=0 \ldots n-j\right)$ ):

$Q 0[j]:=Q:$

$Q 1[j]:=\operatorname{simplify}(\operatorname{subs}(s=1-s, Q 0[j]))$ :

end do:

$>$ \# Constructs the Hermite interpolant in terms of

\# the unknowns $a(1)$ and $b(1)$

$p:=\operatorname{evalf}\left(\operatorname{sum}\left(a[i l]{ }^{\star} Q 0[i 1]+(-1) \wedge i{ }^{\star} b[i l]{ }^{\star} Q 1[i 1], i 1=0 \ldots n\right)\right):$

$>$ \# Solves integral forms for $a(1)$ and $b(1)$

Eq1: =evalf $\left(1+b[1]-a[1]-\exp (1)+\operatorname{int}\left(p^{*} \sin (s), s=0 \ldots 1\right)\right)$ :

Eq $2:=\operatorname{evalf}\left(1-a[1]-\exp (1)+\operatorname{int}\left((1-s){ }^{\star} p^{\star} \sin (s), s=0 \ldots 1\right)\right)$ :

SS:=evalf (solve $(\{\mathrm{Eq} 1, \mathrm{Eq} 2\},\{\mathrm{a}[1], \mathrm{b}[1]\}), 10)$;

$$
S S:=a_{1}=-1.662597558, b_{1}=-0.03929345928
$$

>\# The solution polynomial ps

ps : =simplify (subs $((a[1]=\operatorname{rhs}(\operatorname{sS}[1]), b[1]=\operatorname{rhs}(\operatorname{sS}[2])\}, p))$ :

$>$ \# plot of solution polynomial

plot (ps, s=0..1)

\section{References}

[1] U. M. Ascher, R. M. M. Mattheij and R. D. Russell, Numerical solution of boundary value problems for ordinary different equations (Prentice-Hall, Englewood Cliffs, NJ, 1988).

[2] P. J. Davis, Interpolation and Approximation (Blaisdell, New York, 1963).

[3] L. Fox and I. B. Parker, Chebyshev Polynomials in Numerical Analysis (Oxford University Press, London, 1968). 
[4] R. E. Grundy, "The analysis of initial-boundary-value problems using Hermite interpolation", $J$. Comp. Appl. Math. 154 (2003) 63-95.

[5] R. E. Grundy, "The application of Hermite interpolation to the analysis of non-linear diffusive initial-boundary value problems", IMA J. Appl. Math. 70 (2005) 814-838.

[6] R. E. Grundy, "Polynomial representations for initial-boundary-value problems involving the inviscid Proudman-Johnson equation", Quart. J. Mech. App. Math. 59 (2006) 631-650.

[7] C. Hermite, "Sur la formule d'interpolation de Lagrange", J. Reine Ang. Math. 84 (1878) 70-81.

[8] C. Lanczos, "Trigonometrie interpolation of empirical and analytical functions", J. Math. Phys. 17 (1938) 123-199.

[9] C. Lanczos, Applied Analysis (Pitman, London, 1957).

[10] G. M. Phillips, "Explicit forms for certain Hermite approximations", BIT 13 (1973) 177-180.

[11] S. M. Picken, "Algorithms for the solution of differential equations by the selected points method", Technical report, NPL Mathematics Report 34, 1970.

[12] J. Stoer and R. Bulirsch, Introduction to Numerical Analysis (Springer, New York, 1980). 